\title{
Synthesis and characterization of novel oxygenated CdSe window layer for CdTe thin film solar cells
}

\author{
Zheng Bao ${ }^{\mathrm{a}}$, Longxiao Liu ${ }^{\mathrm{b}}$, Xiaoyan Yang ${ }^{\mathrm{a}}$, Ping Tang ${ }^{\mathrm{a}}$, Ke Yang ${ }^{\mathrm{a}}$, Hongting $\mathrm{Lu}^{\mathrm{a}}$, Siying $\mathrm{He}^{\mathrm{a}}$, \\ Jiyang Liu ${ }^{\mathrm{a}}$, Xiaolan $\mathrm{Liu}^{\mathrm{a}}$, Bing Li ${ }^{\mathrm{a}, *}$ \\ a College of Materials Science and Engineering, Sichuan University, Chengdu 610064, China \\ b College of Physical Science and Technology, Sichuan University, Chengdu 610064, China
}

\section{A R T I C L E I N F O}

\section{Keywords:}

CdSe:O thin film

Pulsed laser deposition

CdSe:O/CdTe solar cell

\begin{abstract}
A B S T R A C T
Significant increases in the solar conversion performance of thin film CdS/CdTe solar cells have been realized in recent years by the inclusion of wider gap materials, notably CdS:O, in place of the CdS window layer. Similarly, use of CdSe in combination with CdTe has given yet further increases in current density due to the formation of $\mathrm{CdTe}_{1-x} \mathrm{Se}_{x}$ and the resulting enhanced long wavelength collection. Here we report the synthesis and properties of oxygenated CdSe films and their influence on device performance when included into CdTe solar cells. The $\mathrm{CdSe}$ :O films were made by pulsed laser deposition in oxygen at pressures of 0.01 and $0.03 \mathrm{~Pa}$. Optical transmission measurements indicated that oxygenation acts to increase transparency in the 400-750 $\mathrm{nm}$ range, and that increased oxygen content acts to increase the apparent absorption threshold slightly. The higher transmission acts to increase the spectral response of CdTe solar cells in the range 350-500 nm compared to CdSe- and CdS-containing control devices. Moreover, the already known advantage of enhanced long wavelength response $(850-910 \mathrm{~nm})$ over that of $\mathrm{CdS} / \mathrm{CdTe}$ is retained for both CdSe and CdSe:O window layers. Hence the use of $\mathrm{CdSe}: \mathrm{O}$ as a window layer is demonstrated to increase the current harvesting of these solar cells by increasing both their long and short wavelength performances. These gains in the short circuit current outweigh small losses in fill factor and open circuit voltage making CdSe:O a valuable possible means to increase the performance of CdTe solar cells.
\end{abstract}

\section{Introduction}

The development of low-cost polycrystalline thin film solar energy materials is important for future energy generation. Cadmium telluride thin film absorbers for photovoltaics are well-known to have the advantages of an ideal direct band gap $E_{\mathrm{g}}(1.45 \mathrm{eV})$, and a high absorption coefficient. Its best known p-n junction partner, the nCdS window layer, belongs to II-VI group of compound semiconductor materials, has a band gap $E_{\mathrm{g}}$ of approximately $2.4 \mathrm{eV}$ and good band line ups with CdTe. Currently, the reported power conversion efficiency of typical CdS/CdTe solar cell has exceeded 20\% [1]. In addition, it is also reported that the open circuit voltage of CdS/CdTe devices has broken the $1 \mathrm{~V}$ barrier without substantial decreases in current through doping the CdTe with a Group V element [2]. However, the CdS/CdTe solar cell has a relatively low current density, but this deficit has been corrected by replacing the CdS with wider gap alternatives, notably oxygenated CdS, i.e. CdS:O. Also, Paudel's group [3] noticed that CdSe/ CdTe solar cells present a higher current density and a higher response from $860 \mathrm{~nm}$ to $910 \mathrm{~nm}$. In contrast to $\mathrm{CdS}$, CdSe has also been recognized as an attractive partner layer material for CdTe because of its lower lattice mismatch of around 6\% with CdTe (compared to CdS) and its higher solubility in the CdTe absorber layer. An efficiency of about $15 \%$ of $\mathrm{CdSe} / \mathrm{CdTe}$ thin film photovoltaic device with an area of $0.08 \mathrm{~cm}^{2}$ has already been demonstrated by University of Toledo [3]. Thus, replacing CdS with CdSe has great potential to fabricate highefficiency CdTe-based solar cells. Nevertheless, CdSe thin film shows a comparatively lower band gap $E_{\mathrm{g}}$ of $1.7 \mathrm{eV}$ [4] compared to CdS, which may be disadvantageous. Many researchers improve the short wavelength response of CdTe solar cells through the conventional method of reducing the thickness of $\mathrm{CdS}$ window layer, but the enhanced short circuit current density is at the cost of losses in voltage and fill factor [5]. Here, a novel attempt to incorporate oxygen in the CdSe thin film is made to improve its properties, and further enhance the photoelectric response of $\mathrm{CdSe} / \mathrm{CdTe}$ solar cell in the short wavelength region (in addition to the already demonstrated long wavelength enhancement). It was found that the photocurrent collection could indeed be increased

\footnotetext{
* Corresponding author.

E-mail address: libing70@126.com (B. Li).
} 


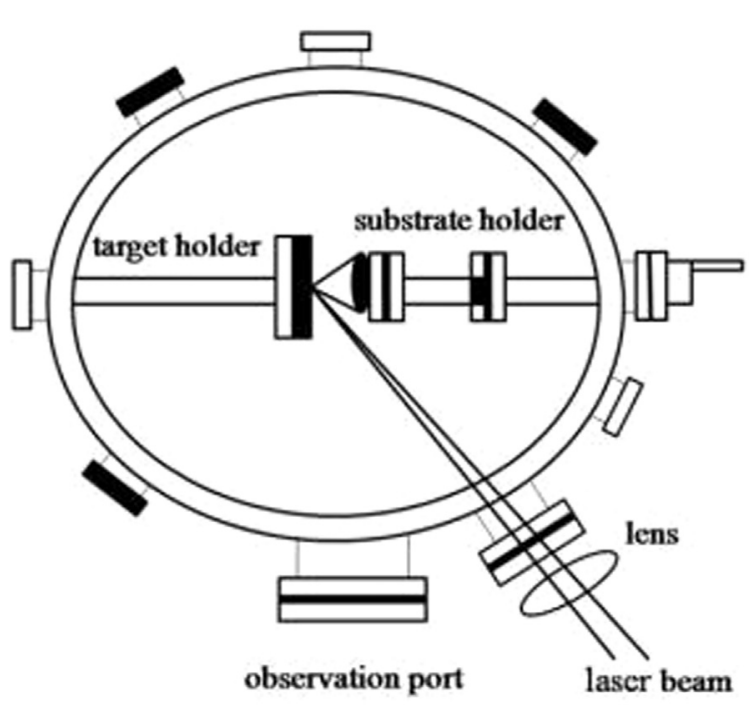

Fig. 1. Schematic diagram of the pulsed laser deposition system.

both in the short and long wavelength ranges compared to standard structures and it is concluded that the use of CdSe:O is viable for CdTebased photovoltaic devices.

It is well-known that there are a large number of techniques available for the formation of CdSe thin films, including chemical bath deposition [6,7], molecular beam epitaxy (MBE) [9], magnetron sputtering $[3,8]$ and close space sublimation (CSS) [10]. In this work, we utilized pulsed laser deposition (PLD) to fabricate both CdSe window layers and oxygenated CdSe window layers. Compared to other methods, pulsed laser deposition can replicate the composition of the targets in the samples easily, and a variety of parameters can be accommodated to optimize the process of manufacture. This is achievable since PLD is able to yield a rapid rise in temperature (> $10^{11} \mathrm{~K} \mathrm{~s}^{-1}$ ), which results in a rapid evaporation of target elements and compounds [11]. In addition, the high surface ablation temperature causes particles arrive on the substrate with an elevated kinetic energy of 1-100 eV, which has the potential to increase the surface mobility of adatoms and is beneficial to manufacture high quality thin films at low substrate temperatures. The instrument also affords accurate computer aided thickness control. It is shown in Fig. 1.

\section{Experimental details}

Four different window layer materials were prepared i.e. CdS, CdSe and CdSe:O having two different amounts of oxygen. For comparative purposes, the thicknesses of all four was kept at $\sim 100 \mathrm{~nm}$, the temperature of substrates was $150{ }^{\circ} \mathrm{C}$ and they were all synthesized by PLD technique. The substrate temperature of $150{ }^{\circ} \mathrm{C}$ was chosen since it had given us the best outcomes in earlier tests in making CdSe/ $\mathrm{CdTe}$ cells. When manufacturing $\mathrm{CdSe}: \mathrm{O}$ thin films, the dynamic oxygen atmosphere was modified from 0 to $0.03 \mathrm{~Pa}$. Photovoltaic devices were synthesized on commercial $\mathrm{SnO}_{2}: \mathrm{F} / \mathrm{SnO}_{2}$ coated soda lime glass substrates (FTO). And FTO glass substrates were cleaned by a series of processes, including with detergent, de-ionized water and isopropyl alcohol. The cleaned FTO glasses were fixed on a heated substrate holder (max. temperature $800^{\circ} \mathrm{C}$ ). Before conducting an experiment, the growth chamber was evacuated to $3.0 \times 10^{-3} \mathrm{~Pa}$ to eliminate air. The $\mathrm{KrF}$ excimer laser $(\lambda=248 \mathrm{~nm}$, pulse duration $20 \mathrm{~ns}$, variable pulse energy from 60 to $150 \mathrm{~mJ}$ ) was used at its optimal energy of $90 \mathrm{~mJ}$ and pulse repetition frequency of $6 \mathrm{~Hz}$ [12-14].

The single thin films were characterized by UV-Vis-NIR spectrophotometer (PerkinElmer Lambda 950), and X-ray diffraction (XRD) (Dan Dong Hao Yuan DX-2700) with using Cu (Ka) $1.54 \AA$ X-rays. The cross-sectional structure of $\mathrm{CdS} / \mathrm{CdTe}$ and $\mathrm{CdSe} / \mathrm{CdTe}$ double layers were measured by scanning electron microscopy (SEM) (Hitachi S4800). The composition of the oxygenated CdSe films was examined by energy dispersive spectrometery (EDS) (Hitachi S-4800). In order to eliminate interferences from the substrate, the compositional analysis was done on films that were specially deposited onto silicon substrates. The surface morphologies of the pure CdSe and the CdSe:O were also investigated by scanning electron microscopy (SEM) (Hitachi S-4800).

For the device fabrication, the CdTe layers were fabricated using the industrially compatible method of close space sublimation (CSS) [15]. CSS employed high-purity CdTe powders as the source material, and the distance of source to substrate was approximately $5 \mathrm{~mm}$. During deposition, the substrate temperature was maintained at $550-580{ }^{\circ} \mathrm{C}$ and the source temperature was $80-100{ }^{\circ} \mathrm{C}$ higher. Although the base chamber pressure was $0.03 \mathrm{KPa}$, we could introduce a mixed gas $\mathrm{Ar}+\mathrm{O}_{2}$ ambient (Ar 90\%, $\left.\mathrm{O}_{2} 10 \%\right)$ at $1.6 \mathrm{KPa}$. Oxygen is thought to increase the acceptor density of CdTe. A CdTe absorber thickness of $5 \mu \mathrm{m}$ was used in the devices. Subsequently, the completed CdTe layer underwent heat treatment at $390{ }^{\circ} \mathrm{C}$ for $35 \mathrm{~min}$ in a tube furnace with $\mathrm{CdCl}_{2}$, which is thought to enable grain growth and passivation hence reducing carrier recombination. After heat treatment the CdTe surface was cleaned by etching, which is thought to form a tellurium-rich layer to enhance the p-conductivity of the CdTe layer for contacting [16,17]. A back contact of $\mathrm{ZnTe}: \mathrm{Cu}(\sim 100 \mathrm{~nm})$ was then deposited by PLD at $180^{\circ} \mathrm{C}$. Then the structure was in-situ annealed under a protective pressure of $2 \times 10^{4} \mathrm{~Pa}$ of $\mathrm{N}_{2}$ at $200{ }^{\circ} \mathrm{C}$ in order to facilitate the $\mathrm{Cu}$ diffusion. Finally, Au electrodes $(\sim 80 \mathrm{~nm}$ thick) were applied by thermal evaporation. The finished device comprised many small cells each having an area of $0.08 \mathrm{~cm}^{2}$. Fig. 2 shows the schematic illustration of the CdTe solar cell device manufactured in this work.

\section{Results and discussion}

\subsection{Optical transmittance and band gaps}

As shown in Fig. 3(a), it could be seen that three window layers displayed different optical transmittance curves. However, both the CdSe and the oxygenated samples show $>80 \%$ transmittance in the long wavelength region. Nevertheless, below $700 \mathrm{~nm}$ the pure CdSe film shows low optical transmittance as expected from its band gap of $1.7 \mathrm{eV}$. Despite this, it is known that when CdSe is included in CdTe photovoltaic devices, dissolution of the CdSe in the CdTe - and the resulting downward bandgap bowing, cause enhanced long wavelength solar cell response, rather than the short wavelength response (or

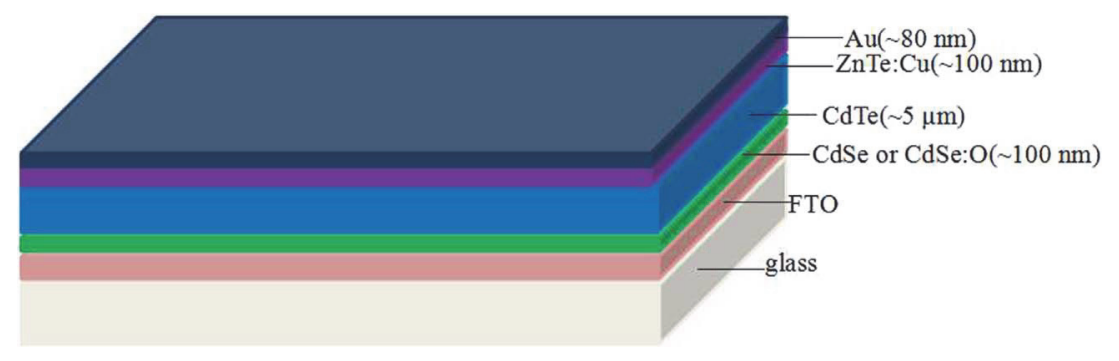

Fig. 2. Schematic illustration of CdTe solar cell device manufactured in this work. 
a

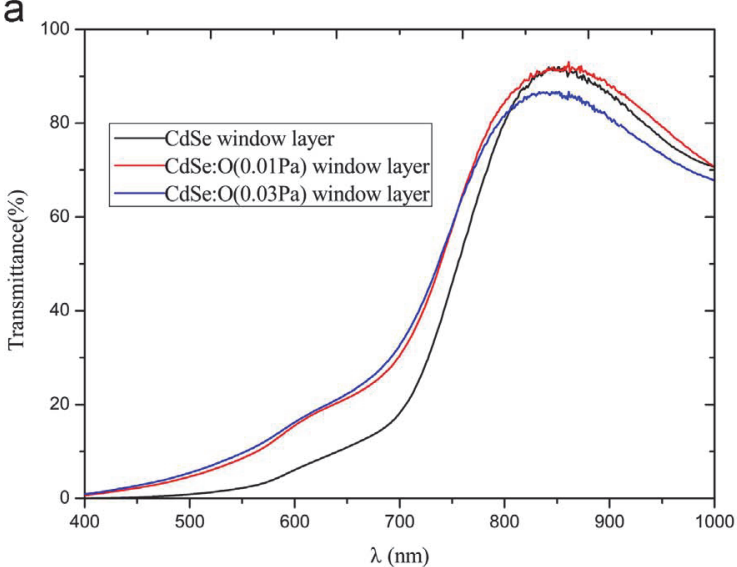

b

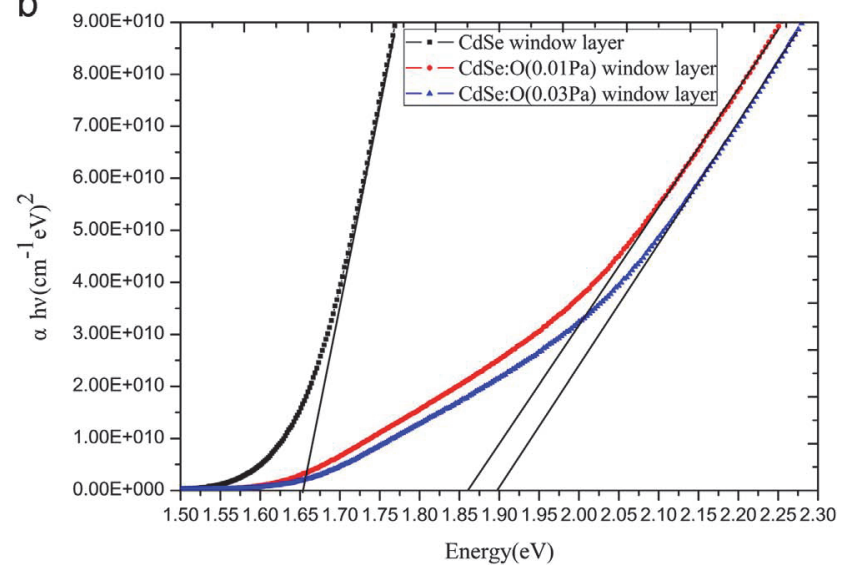

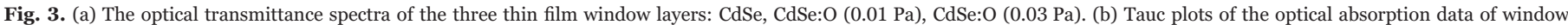

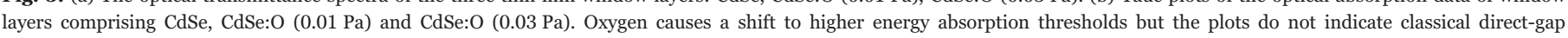
behaviour.

parasitic absorption) that might be expected. This interdiffusion occurs readily during the growth of the CdTe part of the solar cell and its subsequent processing.

However, for the case of oxygenated CdSe:O films [18,19], transmission in the range $400-750 \mathrm{~nm}$ is increased progressively over that for CdSe upon inclusion of higher levels of oxygen from zero to $0.03 \mathrm{~Pa}$, as shown in Fig. 3(a). Moreover, the increase in oxygen in the growth apparatus was accompanied by an apparent blue-shift of the absorption edge i.e. oxygen appears to increase the threshold for optical absorption.

To investigate this further, we made Tauc plots for the three films as shown in Fig. 3(b). While there is a general trend of a shift to higher energy for the onset of absorption with increasing oxygen content, the plots are not linear, and the fits with their intercepts of 1.652, 1.862 and $1.889 \mathrm{eV}$ are made. To conclude that the shift is due to simple modification of the bandgap to higher levels would therefore be tentative. An alternative explanation - that the material is a mixture rather than a solid solution - is also plausible.

\subsection{Crystal structure}

Fig. 4 shows the XRD patterns of the CdSe and CdSe:O thin films deposited at vacuum, 0.01 $\mathrm{Pa}$ oxygen pressure, and $0.03 \mathrm{~Pa} \mathrm{O}_{2}$ pressures. All the films had a clear (111) CdSe peak occurring at $2 \theta=25.35^{\circ}$, $25.45^{\circ}$ and $25.40^{\circ}$ respectively, this being identified by comparison with JCPDS data. Oxygen appears to have had the effect of increasing

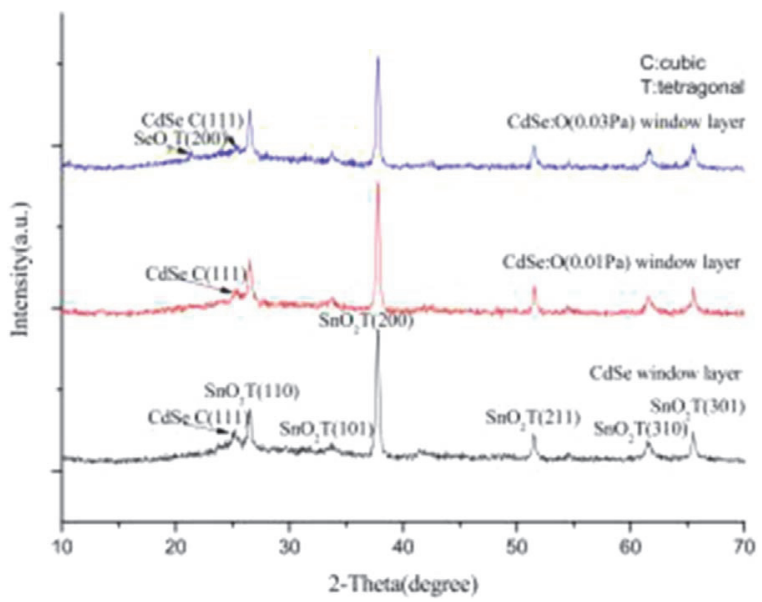

Fig. 4. X-ray diffraction patterns of CdSe and CdSe:O thin films grown under the three conditions. the Bragg angle of the (111) peak, which supports the idea of a solid solution. For all samples, there were weaker peaks that were identified as (220), (311), (400) indicating that all the completed films were polycrystalline regardless of their oxygen content. For films fabricated with the highest level of oxygen $(0.03 \mathrm{~Pa})$ an additional peak that was consistent with being from (200) tetragonal $\mathrm{SeO}_{2}$, was present. It is therefore possible that $\mathrm{CdSe}: \mathrm{O}$ comprises a mixture of $\mathrm{SeO}_{2}$ and a CdSe-rich phase. Finally, since the intensity of the primary (CdSe 111) diffraction peak gradually decreased with the increase of oxygen pressure, it is possible that oxygen suppresses crystallinity, as reported previously in the literature for the case of CdS:O $[20,21]$.

\subsection{Surface morphology and cross-sectional structure}

Scanning electron microscopy (SEM) was utilized to further give more surface morphology information about the CdSe thin films and oxygenated CdSe thin films. CdSe and CdSe:O thin films were fabricated by pulsed laser deposition. The surface of these films was quite compact, and the crystalline size was so small that it was very difficult to distinguish the grain boundary like Fig. 5(a). Therefore, for further investigating the surface morphology of these films, Three thin films fabricated were annealed in dry air ambient at $385^{\circ} \mathrm{C}$ for $30 \mathrm{~min}$. The grains underwent regrowth and became larger than before as shown in Fig. 5b-d. However, from these images, we found there existed several voids, which might form the recombination center to lower the performance of solar cells. Compared to CdSe thin film, the voids of the oxygenated CdSe thin films were relatively fewer, and that of CdSe thin film with $0.01 \mathrm{~Pa}$ oxygen were distinctly fewer than CdSe thin film with $0.03 \mathrm{~Pa}$ oxygen. These studies describe that oxygen plays a significant role in thin films or solar cells.

Next, the microstructure of CdTe in combination with both CdS and CdSe are compared by cross sectional SEM. Care was taken to deposit $100 \mathrm{~nm}$ of each in order to allow meaningful comparison.

It was found that after the deposition of CdTe layer and heat treatment on top of the CdS and CdSe layers, it was clear that the residual CdS layer thickness was greater than that of the CdSe layer. Moreover for the case of CdSe/CdTe double layers, the interface was not clearly visible. To some extent, this confirms that Se has a stronger solubility in CdTe than S. When Se diffuses into the CdTe layer it is expected that the ternary compound $\mathrm{CdTe}_{1-x} \mathrm{Se}_{x}$ forms, and that owing to the bandgap bowing effect, the band gap of the ternary is reduced below that of CdTe, while maintaining a lattice match. These factors are expected to result in an excellent response of $\mathrm{CdSe} / \mathrm{CdTe}$ solar cell in the region of 860-910 $\mathrm{nm}$, realizing the effective collection of photongenerated current in long wavelength $[8,22,23]$ (Fig. 6). 
a

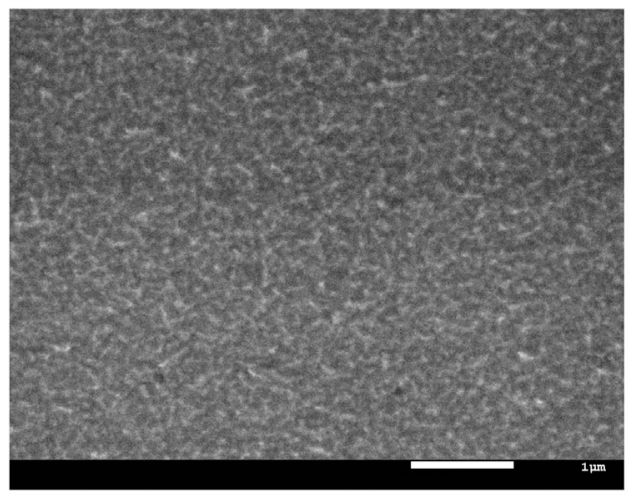

C

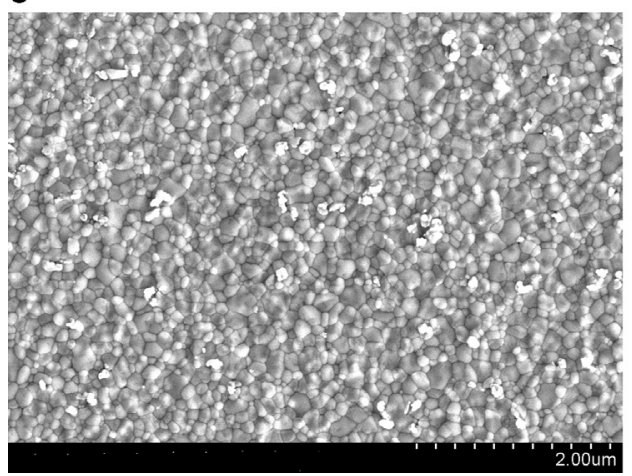

b

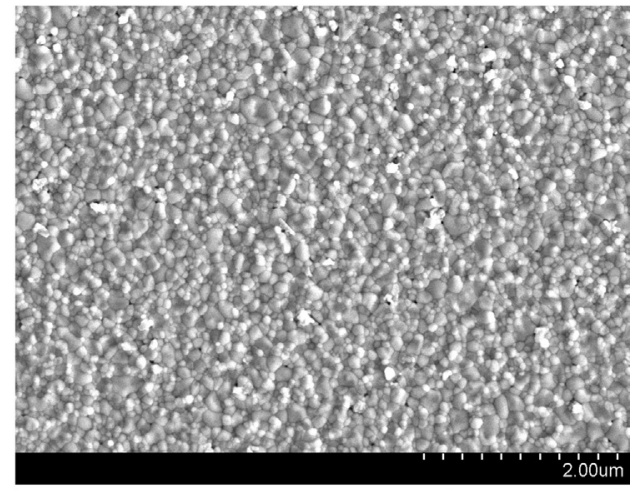

d

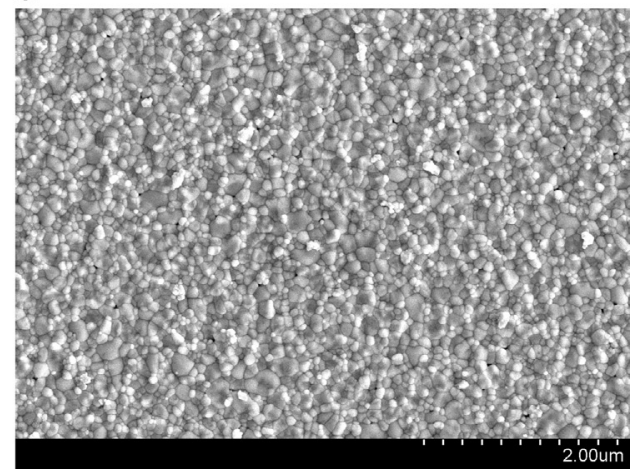

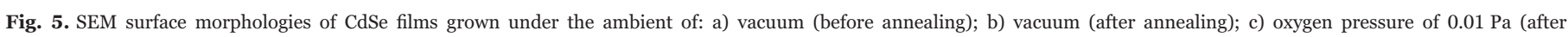
annealing); d) oxygen pressure of $0.03 \mathrm{~Pa}$ (after annealing).

\subsection{Thin film composition analysis}

The EDS technique was used to confirm the composition of the oxygenated CdSe window layers as shown in Fig. 7(a)-b. For the analysis, special samples were grown on silicon wafers in order to avoid interference from the substrate. Due to the intrinsic error of EDS, plus little content of oxygen, the data of the images is not very accurate. However, at least, it indicates that oxygen has been successfully incorporated into the CdSe thin films.

\subsection{Solar cell characteristics}

Following on from the studies of the window layers themselves, a comparative study of CdTe-based solar cells made with the three window layers (CdS, CdSe, and CdSe:O having the two oxygen concentrations) was undertaken to understand their effects on devices. The device structure was: window layer (CdS, CdSe or CdSe:O)(PLD)/ absorber CdTe (CSS)/contact ZnTe:Cu(PLD)/electrode Au (there was no anti-reflection coating). Current - voltage $(J-V)$ measurements were obtained under AM1.5 illumination yielding the efficiency ( $\eta$ ), fill factor $(F F)$, short circuit current density $\left(J_{\text {sc }}\right)$, and open voltage $\left(V_{o c}\right)$ values listed in Table 1, with the best performance for each device being selected for presentation. It could be clearly seen that CdS/CdTe solar cell had the highest open circuit voltage of $713 \mathrm{mV}$. However, it also had the lowest current density $\left(20.1 \mathrm{~mA} / \mathrm{cm}^{2}\right)$ as might be expected from parasitic absorption in the CdS. With a CdSe window layer, an increase of current density $\left(21.8 \mathrm{~mA} / \mathrm{cm}^{2}\right)$ was realized, meanwhile, but at the expense of a much lower voltage of $621 \mathrm{mV}$ compared to that of the CdS/CdTe device (i.e. $92 \mathrm{mV}$ lower). This is presumed to be due to the low bandgap of $\mathrm{CdSe}(1.7 \mathrm{eV})$ compared to $\mathrm{CdS}$ and the possibility that the built-in potential value is small. However, the inclusion of oxygen in the CdSe acted to overcome the limitations of the undoped binary CdSe window layers: When oxygen pressure was $0.01 \mathrm{~Pa}$, the overall efficiency rose to $8.9 \%$, this being driven by an a

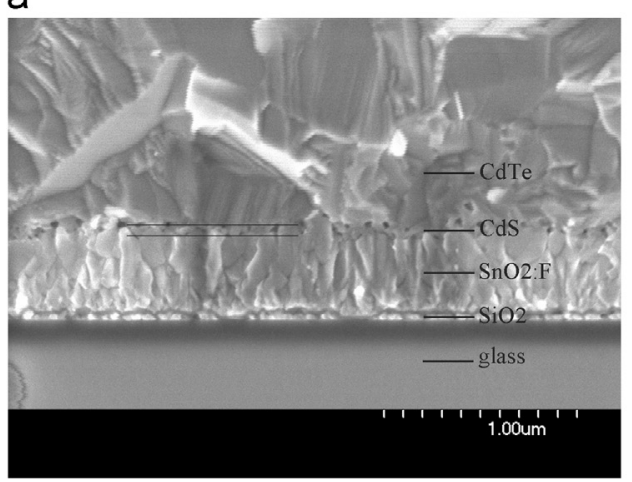

b

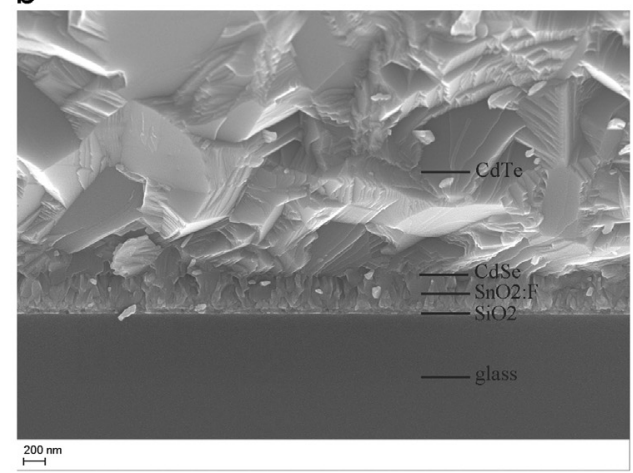

Fig. 6. The cross-sectional SEM images of CdS/CdTe and CdSe/CdTe double layers: a) CdS/CdTe; b) CdSe/CdTe. 
a

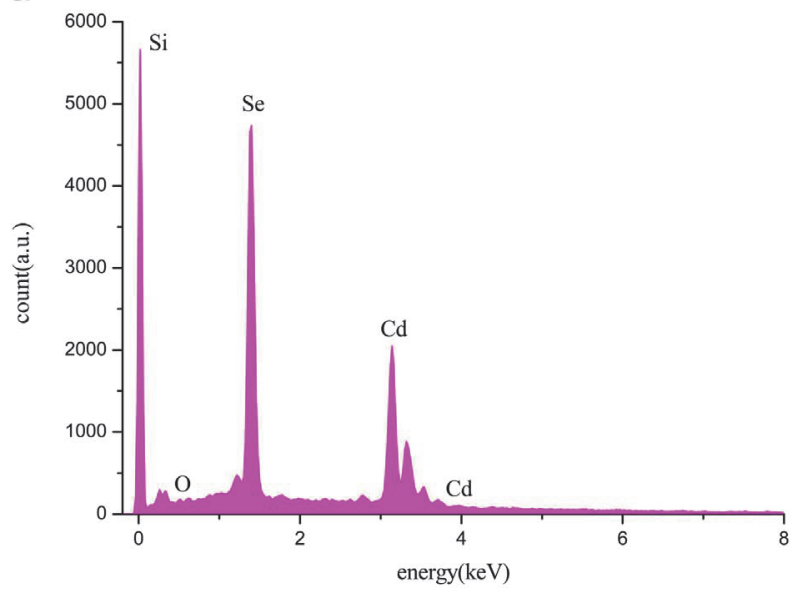

b

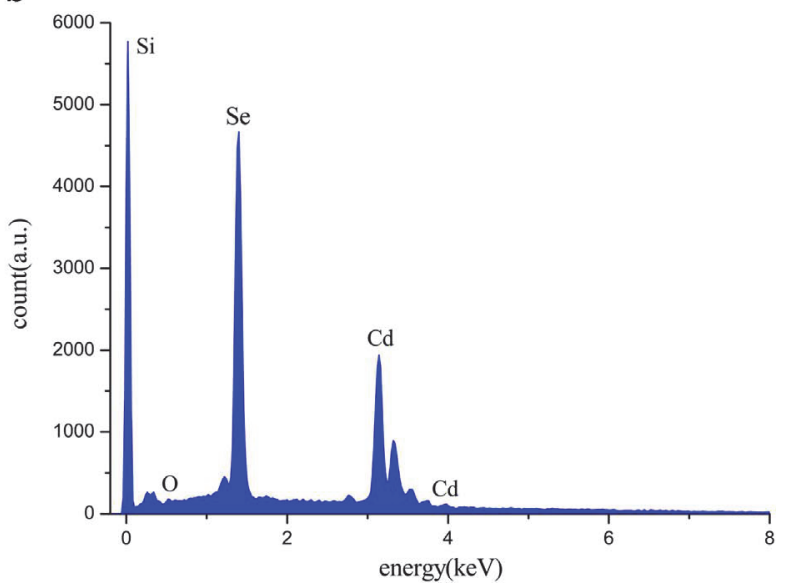

Fig. 7. EDS spectra of oxygenated CdSe:O thin films deposited on silicon wafers: a) CdSe:O (0.01 Pa); b) CdSe:O (0.03 Pa).

Table 1

The best performance of CdS/CdTe solar cells and CdSe/CdTe solar cells with the CdSe window layers prepared by pulsed laser deposition under different deposition temperatures.

\begin{tabular}{lllll}
\hline Window layer & Efficiency (\%) & FF (\%) & $\mathrm{J}_{\mathrm{sc}}\left(\mathrm{mA} / \mathrm{cm}^{2}\right)$ & $\mathrm{V}_{\mathrm{oc}}(\mathrm{mV})$ \\
\hline $\mathrm{CdS}$ & 8.56 & 60.12 & 20.1 & 713 \\
$\mathrm{CdSe}$ & 7.52 & 55.40 & 21.8 & 621 \\
$\mathrm{CdSe}: \mathrm{O}(0.01 \mathrm{~Pa})$ & 8.91 & 58.37 & 22.5 & 678 \\
$\mathrm{CdSe}: \mathrm{O}(0.03 \mathrm{~Pa})$ & 7.60 & 55.01 & 21.5 & 642 \\
\hline
\end{tabular}

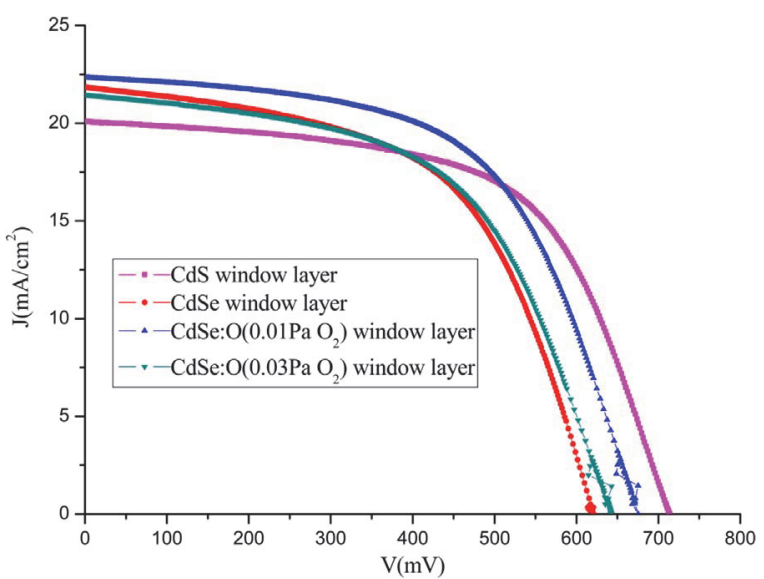

Fig. 8. $J-V$ curves of CdTe-based solar cells with the CdS, CdSe and two CdSe:O window layers.

increased current density $\left(J_{\mathrm{sc}}=22.5 \mathrm{~mA} / \mathrm{cm}^{2}\right)$, and open circuit voltage $\left(V_{o c}=678 \mathrm{mV}\right)$. Nevertheless, further improvements from incorporating more oxygen could not be obtained, and increasing the oxygen pressure during growth to $0.03 \mathrm{~Pa}$ acted to decrease all of the working parameters of the solar cells, including the $J_{\mathrm{sc}}$. Hence it was found that there is an optimum level of oxygen incorporation in $\mathrm{CdSe}: \mathrm{O}$ that gives a maximum in the solar cell performance (Fig. 8).

To further understand the underlying mechanism of this variation, external quantum efficiency (EQE) measurement were made and are shown Fig. 9 for the cells with CdS, CdSe and the two compositions of CdS:O. Firstly the long wavelength response is discussed: While the cell with a CdS window has an EQE threshold at about $850 \mathrm{~nm}$, all of the cells containing either CdSe or CdSe:O showed identical enhanced long wavelength response extending to about $910 \mathrm{~nm}$. This response is consistent with the literature interpretation that CdSe diffuses into the CdTe (during growth and processing), and that bowing [3] causes the bandgap to dip to $910 \mathrm{~nm}$, this being below the intrinsic bulk CdTe

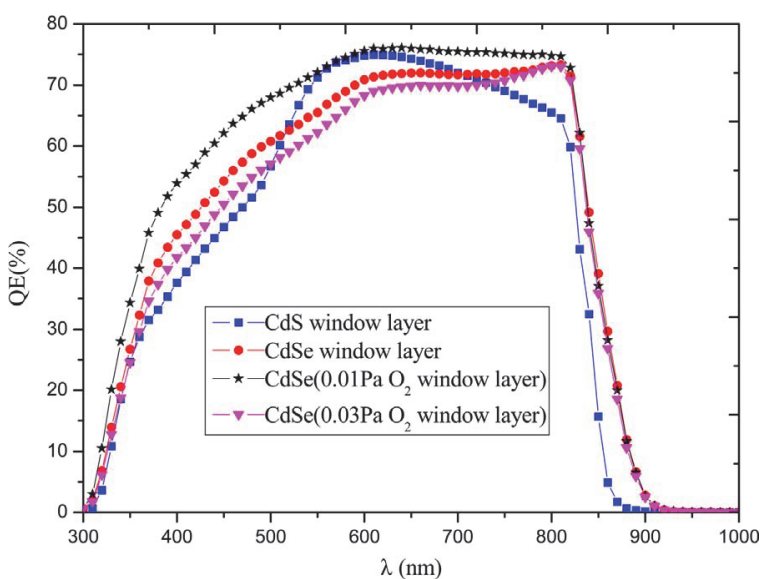

Fig. 9. Measured quantum efficiency spectra of CdTe-based solar cells with different window layers.

value of $860 \mathrm{~nm}$ and hence extending the near infra-red response of the cells. From the coincidence of the lines at the long wavelength side of Fig. 9, this diffusion effect appears to take place identically regardless of the level of oxygenation of the CdSe, and all gave an increased long wavelength response over that of CdS/CdTe.

Secondly the EQE response in the range $350-500 \mathrm{~nm}$ is discussed. This is the region above the band gap of CdS, and which in the CdS/ CdTe device is subject to the parasitic absorption that gives current loss in this wavelength range - unless the CdS is very thin. Indeed, the curve for the device with the CdS window layer shows a step at $\sim 500 \mathrm{~nm}$ that is typical for a CdS-containing device. On the contrary, the devices with CdSe and CdSe:O all had higher EQE in the range 350-500 $\mathrm{nm}$ than the CdS-containing device. For the case of CdSe compared to CdSe:O $\left(0.01 \mathrm{~Pa} \mathrm{O}_{2}\right.$ in deposition), the apparent increase in the band gap of the CdSe:O shown in Fig. 3 gave the expected result that the EQE of the cell with the oxygenated CdSe:O had a higher EQE. However, this expected trend did not continue for the most highly oxygenated window layer tested, namely the CdSe:O $\left(0.03 \mathrm{~Pa} \mathrm{O}_{2}\right.$ in deposition), for which the EQE in the range $350-500 \mathrm{~nm}$ was the lowest of all those in the CdSe and CdSe:O series - and was only just above that of CdS. So paradoxically, even though the CdSe:O sample grown under the most oxygen-rich conditions exhibited the most advantageous optical transmission when grown as a single layer, it gave inferior solar cell performance. We speculate that it is possible that high levels of oxygen incorporation in CdSe introduces high levels of point or cluster defects, either within the CdSe:O films, or at the interfaces of the CdSe:O/CdTe structures. This might act to cause 
degradation of the electrical junction performance or junction position that would degrade the EQE in the region $350-500 \mathrm{~nm}$.

Lastly we note that the substitution of CdS with CdSe or any of the CdSe:O compositions tried acts to degrade the fill factor from about 60 to about $55 \%$, except in the case of CdSe:O $\left(0.01 \mathrm{~Pa} \mathrm{O}_{2}\right)$ for which the degradation was less severe. Fill factor loss is normally associated with current leakage paths or incomplete film coverage, although that was not investigated here.

Overall though, the inclusion of $\mathrm{CdSe}$, and $\mathrm{CdSe}: \mathrm{O}$ as a replacement window layer for $\mathrm{CdS}$ in CdTe solar cells - especially at the composition obtained with $0.01 \mathrm{~Pa}$ of $\mathrm{O}_{2}$ during growth - acted to increase the solar conversion efficiency. Although there was a slight loss of $V_{o c}$ and FF, CdSe:O acted to increase the EQE response in both a) the low (350$500 \mathrm{~nm})$ and b) the high (850-910 nm) ranges over and above that achieved with CdS. This is considered to be due to a) the increase in short wavelength optical transmission in $\mathrm{CdSe}: \mathrm{O}$ and b) the increase in long wavelength absorption by $\mathrm{CdTe}_{1-x} \mathrm{Se}_{x}$. These increase in optical performance outweighed the voltage and fill factor decreases. Nevertheless, since excess oxygen reverses the device benefits, in our later work, we would continue to search for the most optimum content of oxygen incorporation and optimize all kinds of preparation conditions to further improve the performance of CdSe/CdTe solar cells. Moreover, the problem of the reduced fill factor that is present in all $\mathrm{CdSe} / \mathrm{CdTe}$ and $\mathrm{CdSe}: \mathrm{O} / \mathrm{CdTe}$ solar cells remains, and this might be related to the intrinsic properties of semiconductor materials - hence it may become the focus of subsequent research. All in all, we demonstrated the effectiveness of PLD in a totally novel study of deposition CdSe:O window layer by ablation in an oxygen ambient. We believe that this effective method and window layer chemistry can help promote the development of CdTe-based solar cells.

\section{Conclusions}

In this study we have exploited the reproducibility and reliability of pulsed laser deposition in a novel study of both CdSe:O films and CdTe photovoltaic devices made using them as window layers - as alternatives to CdS and plain CdSe. After annealing, on the surface the PLD CdSe:O films showed fewer voids than that of pure CdSe thin films. The higher levels of oxygen caused the XRD peaks of CdSe to become less strong, presumably due to amorphisation caused by the oxygen. The most highly oxygenated samples showed evidence of $\mathrm{SeO}_{2}$ in their XRD spectra. Increasing the oxygen pressure during growth also acted to increase the short wavelength optical transmission and shifted the apparent absorption edge to higher energies in the approximate range 1.65-1.89 eV. The result that CdSe interdiffuses into CdTe faster than does CdS was confirmed in a comparative diffusion experiment. Likefor-like solar cell devices containing window layers of CdS, CdSe, CdSe (grown under $0.01 \mathrm{~Pa} \mathrm{O}_{2}$ ) and $\mathrm{CdSe}$ (grown under $0.03 \mathrm{~Pa}_{2} \mathrm{O}_{2}$ ) demonstrated that: a) the long wavelength $\mathrm{EQE}$ increases that are ascribed to bandgap-bowed interdiffused layers of $\mathrm{CdTe}_{1-x} \mathrm{Se}_{x}$. are obtained for both plain CdSe and all compositions of CdSe:O tested, and b) the increase in short wavelength optical transmission observed for CdSe:O (over that of CdSe) is responsible for increased EQE response in solar cells having CdSe:O window layers over and above those with CdS or plain CdSe. There is an optimum for the level of oxygen in the CdSe:O that gives the best cells and this acts to increase the EQE in both the low $(350-500 \mathrm{~nm})$ and long $(850-910 \mathrm{~nm})$ wavelength ranges. Overall this acts to increase the solar cell efficiency, despite some losses in $\left(V_{o c}\right)$ and fill factor. Further work will focus on identifying the optimum composition for CdSe:O more exactly and investigating the reasons why higher levels of oxygen cause the cell performance to decline.

\section{Acknowledgements}

This work was supported by the National Science Foundation of China (grant: 61574094) and matching support from the State of Kansas via Kansas Technology Enterprise Corporation.

\section{References}

[1] See $\langle$ http://investor.firstsolar.com/releasedetail.cfm? $\rangle$ Release ID $=828273$ for First Solar press release.

[2] J.M. Burst, J.N. Duenow, D.S. Albin, CdTe solar cells with open-circuit voltage breaking the $1 \mathrm{~V}$ barrier, Nat. Energy 15 (2016) 1-7.

[3] N.R. Paudel, Y. Yan, Enhancing the photo- currents of CdTe thin-film solar cells in both short and long wavelength regions, Appl. Phys. Lett. 105 (2014) 183510.

[4] A.V. Kokate, U.B. Suryavanshi, C.H. Bhosale, Structural, compositional, and optical properties of electrochemically deposited stoichiometric CdSe thin films from nonaqueous bath, in: Proceedings of International Symposium on Solar Cells and Solar Energy Materials held at the Mexican-Academy-of-Materials-Science Annual Meeting, 80, 2006, pp. 156-160.

[5] K. Nakamura, M. Gotoh, T. Fujihara, Influence of CdS window layer on 2- $\mu$ m thick CdS/CdTe thin film solar cells, Sol. Energy Mater. Sol. Cells 75 (2003) 185.

[6] M. Simurda, P. Nemec, Morphology of CdSe films prepared by chemical bath deposition: the role of substrate, in: Proceedings of Symposium on Thin Film and Nanostructured Materials for Photovoltaics held at the 2005 EMRS Meeting, 511, 2005, pp. 71-75.

[7] P.P. Hankare, S.D. Delekar, M.R. Asabe, P.A. Chate, Synthesis of cadmium selenide thin films at low-temperature by simple chemical route and their characterization, Phys. Chem. Solids 67 (2006) 2506.

[8] N.R. Paudel, Y. Yan, Current enhancement of CdTe-based solar cells, IEEE J. Photovolt. 5 (2015) 1492-1496.

[9] S. Sorokin, T. Shubina, Peculiarities of migration-enhanced-epitaxy (MEE) versus molecular beam epitaxy (MBE) growth kinetics of CdSe fractional monolayers in ZnSe, in: Proceedings of the 10th International Conference on Molecular Beam Epitaxy (MBE-X), 201, 1999, pp. 461-464.

[10] M.M. Ivashchenko, Morphological, structural, compositional properties and IRspectroscopy of CdSe films deposited by close-spaced vacuum sublimation Opanasyuk A S, Vacuum 119 (2015) 81-87.

[11] B. Li, J. Liu, G. Xu, R. Lu, L. Feng, J. Wu, Development of pulsed laser deposition for CdS/CdTe thin film solar cell, Appl. Phys. Lett. 101 (2012) 153903.

[12] Chao Ding, Zhenxun Ming, Bing Li, Preparation and characterization of pulsed laser deposited CdTe thin films at higher FTO substrate temperature and in $\mathrm{Ar}+\mathrm{O}-2$ atm, Mater. Sci. Eng. B 178 (2013) 801-806.

[13] Zhengxun Ming, Chao Ding, Bing Li, Study of Ar+O-2 deposition pressures on properties of pulsed laser deposited CdTe thin films at high substrate temperature, J. Mater. Sci.: Mater. Electron. 25 (2014) 1901-1907.

[14] Run Luo, Bo Liu, Bing Li, The large-area CdTe thin film for CdS/CdTe solar cell prepared by physical vapor deposition in medium pressure, Appl. Surf. Sci. 360 (2016) 744-748

[15] J. Schaffner, M. Motzko, $12 \%$ efficient CdTe/CdS thin film solar cells deposited by low-temperature close space sublimation, J. Appl. Phys. 110 (2011) 064528.

[16] A. Rios-Flores, J. Pena, A study of vapor CdCl2 treatment by CSS in CdS/CdTe solar cells, Sol. Energy 84 (2010) 1020-1026.

[17] K.M. AbuEl-Rub, S. Tair, Effects of $\mathrm{CdCl} 2$ heat treatment on the morphological and chemical properties of CdTe/CdS thin films solar cells, Appl. Surf. Sci. 258 (2012) 6142-6147.

[18] D.M. Meysing, C.A. Wolden, Properties of reactively sputtered oxygenated cadmium sulfide (CdS:O) and their impact on CdTe solar cell performance, J. Vac. Sci. Technol. A 33 (2015) 0212031-0212037.

[19] N.R. Paudel, C.R. Grice, The effects of high temperature processing on the structural and optical properties of oxygenated CdS window layers in CdTe solar cells, J. Appl. Phys. 116 (2014) 044506.

[20] M.M. Junda, C.R. Grice, Effects of oxygen partial pressure, deposition temperature, and annealing on the optical response of CdS:O thin films as studied by spectroscopic ellipsometry, J. Appl. Phys. 120 (2016) 015306.

[21] M.A. Islam, M.U. Khandaker, Effect of deposition power in fabrication of highly efficient CdS:O/CdTe thin film solar cell by the magnetron sputtering technique, Mater. Sci. Semicond. Process. 40 (2015) 90-98.

[22] D.U. Kim, C.M. Hangarter, R. Debnath, Backcontact CdSe/CdTe windowless solar cells, Sol. Energy Mater. Sol. Cells 109 (2013) 246-253.

[23] J.D. Poplawsky, Guo Wei, Structural and compositional dependence of the CdTexSe1-x alloy layer photoactivity in CdTe-based solar cells, Nat. Commun. 7 (2016) 12537. 